\title{
On the Uplink Traffic Distribution in Time for Duty-cycle Constrained LoRaWAN Networks
}

\author{
Konstantin Mikhaylov \\ Centre for Wireless Communications \\ University of Oulu \\ Oulu, Finland \\ konstantin.mikhaylov@oulu.fi
}

\begin{abstract}
In the recent years, the LoRaWAN Low Power Wide Area Network (LPWAN) technology became a critical connectivity enabler for many Internet of Things (IoT) grade monitoring applications and has attracted substantial attention from Academy, Industry, and businesses. One of the most widespread assumption relative to the LoRaWAN and the basis for many studies has been the uniformity of distribution in time of uplink packet transmissions by the different machine devices composing the network. However, recent experimental studies revealed that this implication does not always hold in real-life networks composed of multiple devices, which operate under duty-cycle restrictions. This study dives deeper to identify the reason underlying this effect, which can potentially create negative consequences for the performance of the whole network. Specifically, the paper starts by detailing the key aspects of LoRaWAN procedures and mechanisms and hypothesize that non-uniform distribution of UL transmissions may be caused by a cumulative effect of (i) over-the-air activation (OTAA) procedure, (ii) duty-cycle restrictions and their implementation, and (iii) periodic UL traffic. This hypothesis is validated and confirmed through simulations using the specially-developed model, which captures the details of OTAA and subsequent data transmissions in LoRaWAN. After demonstrating this effect and validity of our hypothesis, several approaches, which can enable to mitigate it, are identified and pinpointed.
\end{abstract}

Index Terms-LoRaWAN, LoRa, LPWAN, Time, Distribution, Traffic, Uniform, Non-uniform, Experiment, Simulation, Performance.

\section{INTRODUCTION}

The evolution of wireless connectivity technologies during recent years has been robust, being driven by the everincreasing demand of the versatile Internet of Things (IoT) applications hitting the market. One of the most notable changes has happened due to the introduction of the Low Power Wide Area Network (LPWAN) class of radio access technologies, which provide energy and cost-efficient wireless connectivity for massive deployments of versatile machine-devices. These technologies gave a significant push for developing novel monitoring applications for Smart City and logistics, environment monitoring and Industry domains. Among several LPWANgrade technologies, the Long Range Wide Area Network (LoRaWAN) became one of the most widely used overall and the most widespread technology operating in the license-free spectrum [1]. This has attracted attention to this technology from Industry, business and Academy.

Significant research efforts have been invested in understanding and dozens of papers have been published focusing on the performance limits and suitability of LoRaWAN for specific applications using analytical, modelling and experimental methods. Some improvements have also been proposed for enhancing reliability, latency, throughput and energy performance of LoRaWAN. However, all the studies known to the authors, base on the fundamental assumption of uniformity of uplink (UL) packet traffic distribution in time from the different devices composing the network. Nonetheless, in our earlier experimental study [2] of a Smart Campus LoRaWAN composing over 300 real-life sensors, we have observed (Fig. 6 in [2]) significant and periodic fluctuation in time of the number of devices attempting to transmit their uplink data. This has resulted in additional packet losses during the most "crowded" periods. To dive deeper into this issue and find the reason for this effect, in this paper, we start by revisiting the basic procedures and their implementation in the conventional LoRaWANs. Following this, we identify and then validate through simulations one potential reason which could cause such behaviour - the cumulative effect of the Over-the-Air Activation (OTAA) procedure and duty-cycle (DC) limitation.

The primary contributions of this study are:

- We demonstrate that the state-of-the-art OTAA and DC accounting mechanisms employed in LoRaWAN may result in non-uniform distribution of UL packets even if the attempts to join ("activate in") the network by the different devices are distributed uniformly in time. Specifically, this is likely to happen if many devices attempt to join the network within a short time.

- We investigate how the different parameters affect the traffic distribution and the performance of the network.

- We suggest several mechanisms, which can potentially mitigate the identified problem by making the distribution of the UL packets more uniform.

In addition to these, the paper also introduces and delivers to the community the designed by us MATLAB simulation model [3] focusing specifically on simulating OTAA and subsequent communication in LoRaWAN to enable validation of our results and the further studies of this issue. This is worth noting that: (i) the OTAA procedure is one of the two and the most secure activation procedure specified for LoRaWAN; (ii) today the LoRaWAN is primarily employed for versatile monitoring applications, many of which measure and transmit their data periodically; (iii) the described scenario of multiple devices 
requiring to join the network may happen not only during initial deployments but also after temporal unavailability (e.g., a firmware update or reboot) of the LoRaWAN GW. This makes the problem, raised and investigated in this study, very relevant as of today.

The paper is organized as follows. In Section II the relevant aspects of the LoRaWAN specification, limitations imposed on LoRaWAN in the EU, and the practical mechanisms employed in real-life devices to address these are discussed. Next, in Section III we identify the pre-conditions and hypothesize how all these mechanisms may result in the UL traffic distribution becoming non-uniform in time. In Section IV we check our hypothesis - first, we present, detail and validate our simulation model, and then provide some illustrative numerical results obtained using it. Finally, Section V concludes the paper, summarizes the results and suggests several mechanisms, which can potentially help to mitigate the identified problem.

\section{TeChNicAl BACKGROUND: LoRAWAN ACTIVATION AND COMMUNiCATION PROCEDURES}

To understand the background of the problem, focused in this study, this is worth recalling the specifics of the LoRaWAN media access protocol and details of LoRaWAN device operation. In the following subsections, we discuss in sufficient details the key aspects relevant for this study, while a more comprehensive information an interested reader can obtain from LoRaWAN specification documents (i.e., [4]) and other relevant literature (e.g., [5]).

\section{A. Basic LoRaWAN Procedures}

By its design, a LoRaWAN network is intended primarily for conveying the UL traffic. The end devices (EDs), whenever they have data, send them in UL to a gateway (GW), which forwards data further to the network server (NS) managing the network. Even though the LoRaWAN specification defines several ED classes, differing primarily for their energy consumption and latency in downlink (DL) access, class A is obligatory and default for all EDs.

An ED implementing class A can send its UL data at any moment. For this, the ED randomly selects one of the supported by the network frequency channels (whilst obeying the respective DC limitation, as discussed in more details further in Subsection II-C). After completing its UL transmission, after the pre-specified delays, an ED opens up to two receive windows (RX1 and RX2, respectively), during which the network can reach this device. A conventional transmit sequence of a class A LoRaWAN ED, and the respective timings are illustrated in Fig. 1. This is worth noting that RX1 is opened in the same frequency channel, that has been used for UL, while RX2 is typically allocated in a dedicated frequency channel, which does not overlap with the channels employed for UL data transfers.

The LoRa modulation, serving as a primary physical (PHY) layer of LoRaWAN, is a variant of a frequency chirp spread spectrum M-ary digital modulation, in which the instantaneous frequency is linearly increased, and then wrapped to the

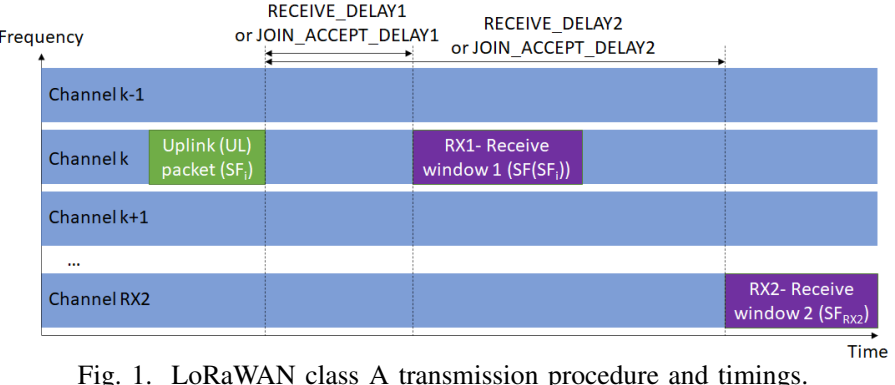

Fig. 1. LoRaWAN class A transmission procedure and timings.

minimum frequency when reaching the maximum frequency of the occupied band [6]. One of the LoRa modulation's key parameters is the spreading factor (SF), which determines the number of chips per symbol and is inversely proportional to the modulation rate of the chirp [7]. By increasing the SF, the transmitter increases the time on-air, raising the energy consumption and reducing the data rate, but boosts the maximum possible communication range. Notably, the signals with different SFs are quasi-orthogonal, allowing transmissions with different SFs being correctly received simultaneously under some pre-conditions [7]. The SF can be allocated to an ED statically, or dynamically adjusted by the network by executing a specialised adaptive data rate (ADR) procedure [8]. The on-air time for LoRaWAN packets can be calculated using equations and parameters from [4], [9], [10] listed below, where $T_{\text {symbol }}, T_{\text {preamble }}$, and $T_{\text {payload }}$ denote the duration of one symbol, packet preamble and packet payload with headers.

$$
\begin{gathered}
T_{\text {symbol }}(S F)=\frac{2^{S F}}{B W} \quad[\mathrm{~s}] \\
T_{\text {preamble }}(S F)=\left(L_{\text {preamble }}+4.25\right) \cdot T_{\text {symbol }}(S F) \\
L_{\text {payload }}=8+\left[\frac{(2 B-S F+7+4 C R C+5 H)}{(S F-2 D E)}\right] \cdot(C R+4) \\
T_{\text {payload }}(S F)=L_{\text {payload }} \cdot T_{\text {symbol }}(S F) \\
T o A(S F)=T_{\text {preamble }}(S F)+T_{\text {payload }}(S F)
\end{gathered}
$$

Here $B W$ is signal bandwidth in $\mathrm{Hz}, B$ is the payload (i.e., PHY_payload) in bytes, $D E$ is 1 for SF11 and SF12 and 0 otherwise, $C R$ denotes the coding rate, $C R C$ denotes the presence of PHY layer cyclic redundancy code ( 1 for UL, 0 for DL), $H$ denotes the presence of the explicit header (for LoRaWAN $H=1$ ), $L_{\text {preamble }}$ and $L_{\text {payload }}$ are the lengths of the preamble and the payload in symbols, respectively. For LoRaWAN operating in the EU frequency bands, according to [4], [11], $C R=1$ and $L_{\text {preamble }}=8$.

\section{B. Activation procedures and packet format}

The operation of any LoRaWAN ED can be subdivided into two phases: (i) activation and (ii) actual data communication. The activation procedure is required to introduce an ED to the network and equip it with all the needed credentials (namely, the device address - DevAddr, the triplet of network session keys (i.e., NwkSEncKey/SNwkSIntKey/FNwkSIntKey), and the application session key (AppSKey) allowing a device to 


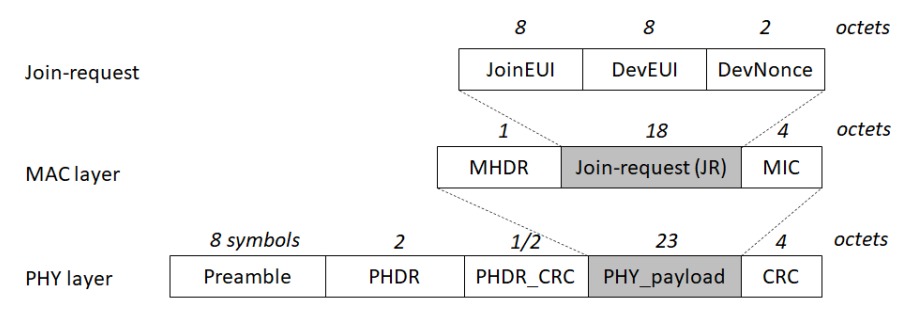

Fig. 2. Format of LoRaWAN Join-request frame.

Join-accept

MAClayer

PHY layer

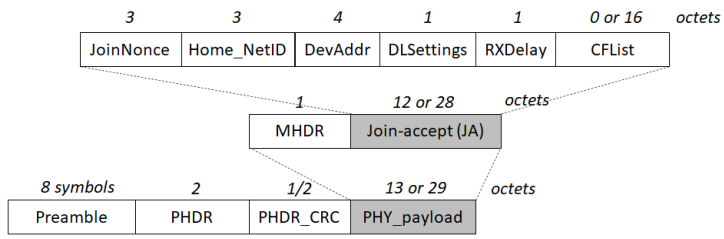

Fig. 3. Format of LoRaWAN Join-accept frame.

operate in the network [11]. According to the LoRaWAN specification [11], activation can be carried in two ways.

The former option is the so-called Activation by Personalisation (ABP) implies that all the needed keys and credentials are provided offline (e.g., flashed to the memory of an ED over a wired or NFC interface). An ABP-activated device can start communication in the network right away after power up with all the needed keys. However, the main downside of ABP is the lack of possibility to change the keys, even if these get compromised, throughout the device's lifetime, except through a manual reprogramming [12].

The alternative approach, named OTAA, implies generating a new set of keys for every new communication session and is thus considered to provide a higher level of security [12]. For this reason, OTAA is the recommended activation method in many deployed LoRaWAN networks. The OTAA procedure implies exchanging two messages, which are referred to as Join-request(JR) and Join-accept(JA). The format of these two messages and that of a generic UL data packet are depicted in Figs. 2-4. Note, that as per LoRaWAN specification, the DL packets do not include the CRC at the PHY layer. This is also worth noting that the JA packet may optionally include the channel list (CFList field) containing the additional UL channels supported by the network. Therefore, the size of the packet payload for these three types of packets is given by: $B_{\mathrm{JR}}=27, B_{\mathrm{JA}(\text { noCFList })}=13, B_{\mathrm{JA}(\text { CFList })}=29$ and

$$
B_{\text {Data }}=16+\operatorname{sign}\left(B_{\mathrm{App}}\right)+B_{\mathrm{App}} \quad[\text { byte }]
$$

where $B_{\mathrm{App}}$ is the application-layer payload in bytes (note, that the maximum possible payload varies depending on the $\mathrm{SF}$ used). The $\operatorname{sign}()$ function takes the value of 0 in case $B_{\mathrm{App}}=0$ and 1 if $B_{\mathrm{App}}>0$ and accounts for the need of frame port (FPort) field presence in case the payload is present. Note, that (6) addresses the case when the optional frame options (i.e., FOpts, which are employed for MAC commands) field is not included in the packet. Also, in the case of conventional UL data packets, the time from the end of a UL transmission to RX1 and RX2 is defined by parameters $R E C E I V E \_D E L A Y 1$ and RECEIVE_DELAY2. These parameters have the recommended default values of $1 \mathrm{~s}$ and 2
TABLE I

LORAWAN EU FREQUENCY SUBBANDS AND RESTRICTIONS [13], [14]

\begin{tabular}{|c|c|c|c|}
\hline Subband [14] & Frequency band, MHz & max DC, \% & max power, $\mathrm{mW}$ \\
\hline h1.3 & $863-865$ & 0.1 & 25 \\
h1.4 & $865-868$ & 1 & 25 \\
h1.5 & $868-868.6$ & 1 & 25 \\
h1.6 & $868.7-869.2$ & 0.1 & 25 \\
h1.7 & $869.4-869.65$ & 10 & 500 \\
h1.9 & $869.7-870$ & 1 & 25 \\
\hline
\end{tabular}

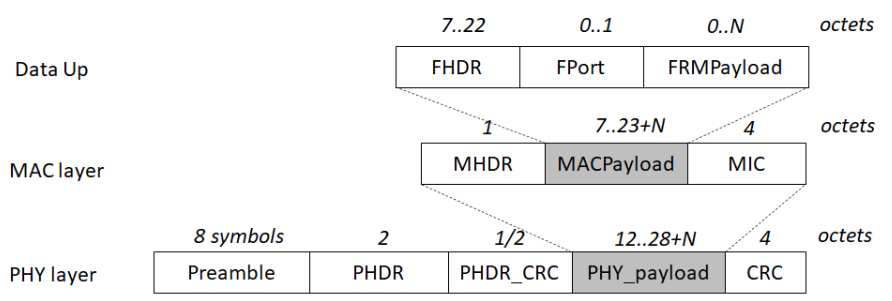

Fig. 4. Format of LoRaWAN uplink data frame.

$\mathrm{s}$, respectively. During the OTAA procedure, instead of these two parameters, the delays JOIN_ACCEPT_DELAY1 and JOIN_ACCEPT_DELAY2, having recommended values of $5 \mathrm{~s}$ and $6 \mathrm{~s}$, respectively, are utilised.

\section{Frequency Regulations and Duty-Cycle}

Since LoRaWAN standard does not imply the obligatory use of a listen-before-talk (LBT), according to the regulations on the frequency use imposed by the respective authorities (i.e., [14] in EU), it has to obey the specified DC restrictions. Following the discussion in [13], [15] in the EU, the LoRaWAN devices are typically classified as short-range devices. The recommended (in [13]) frequency channels for LoRaWAN operation in EU and the respective DC restrictions are summarized in Table I. Note, that LoRaWAN regional parameters document [4] prescribes each device and network to implement the three default LoRaWAN channels (centred at $868.1 \mathrm{MHz}, 868.3 \mathrm{MHz}$ and $868.5 \mathrm{MHz}$ thus belonging to subband h1.5), which should also be used for activation. This is worth noting that due to the high DC and maximum transmit power allowance, a channel in subband h1.7 is often reserved in LoRaWAN networks for DL communication within the second receive window (RX2) [11], [13].

This is worth noting, that while the latest versions (i.e., 1.1) of the LoRaWAN specification do not explicitly specify how DC procedures should be implemented, the earlier versions (e.g., 1.0) explicitly require an ED not to transmit on a channel in the same subband for

$$
T_{\text {backoff }}=\frac{T o A}{D C}-T o A \quad[\mathrm{~s}]
$$

after it has sent a packet in this subband with on-air time ToA, where $D C$ is the maximum DC permitted for this subband. The advantage of this approach is the simplicity of its practical implementation, which has made it popular for many commercial LoRaWAN EDs and GWs.

The on-air times for the JR and JA packets (both when CFList is present and when it is absent) calculated from 5 for different SFs are illustrated in Table II. The table also lists 
TABLE II

ON-AIR AND BACKOFF TIME FOR JA AND JR

\begin{tabular}{|c|c|c|c|c|c|c|}
\hline \multirow[t]{2}{*}{ Parameter } & \multicolumn{6}{|c|}{ Time, $\mathrm{s}$} \\
\hline & SF7 & SF8 & SF9 & SF10 & SF11 & SF12 \\
\hline \multicolumn{7}{|c|}{ On-air time,s } \\
\hline JR & 0.06 & 0.11 & 0.21 & 0.37 & 0.82 & 1.48 \\
\hline JA no CFL & 0.04 & 0.08 & 0.14 & 0.29 & 0.58 & 1.16 \\
\hline JA CFL & 0.07 & 0.12 & 0.23 & 0.41 & 0.82 & 1.64 \\
\hline \multicolumn{7}{|c|}{ Backoff time, $\mathrm{s}(\mathrm{DC}=\mathbf{0 . 1 \%})$} \\
\hline JR & 61.67 & 113.15 & 205.82 & 370.69 & 823.30 & 1482.75 \\
\hline JA no CFL & 46.29 & 82.35 & 164.70 & 288.48 & 576.96 & 1153.92 \\
\hline JA CFL & 66.75 & 123.27 & 226.08 & 411.24 & 904.31 & 1644.95 \\
\hline \multicolumn{7}{|c|}{ Backoff time, s $(\mathrm{DC}=1 \%)$} \\
\hline JR & 6.17 & 11.32 & 20.58 & 37.07 & 82.33 & 148.28 \\
\hline JA no CFL & 4.59 & 8.16 & 16.32 & 28.59 & 57.18 & 114.35 \\
\hline JA CFL & 6.62 & 12.22 & 22.40 & 40.75 & 89.62 & 163.01 \\
\hline \multicolumn{7}{|c|}{ Backoff time, s $(\mathrm{DC}=10 \%)$} \\
\hline JR & 0.62 & 1.13 & 2.06 & 3.71 & 8.23 & 14.83 \\
\hline JA no CFL & 0.42 & 0.74 & 1.48 & 2.60 & 5.20 & 10.40 \\
\hline JA CFL & 0.60 & 1.11 & 2.04 & 3.71 & 8.15 & 14.82 \\
\hline
\end{tabular}

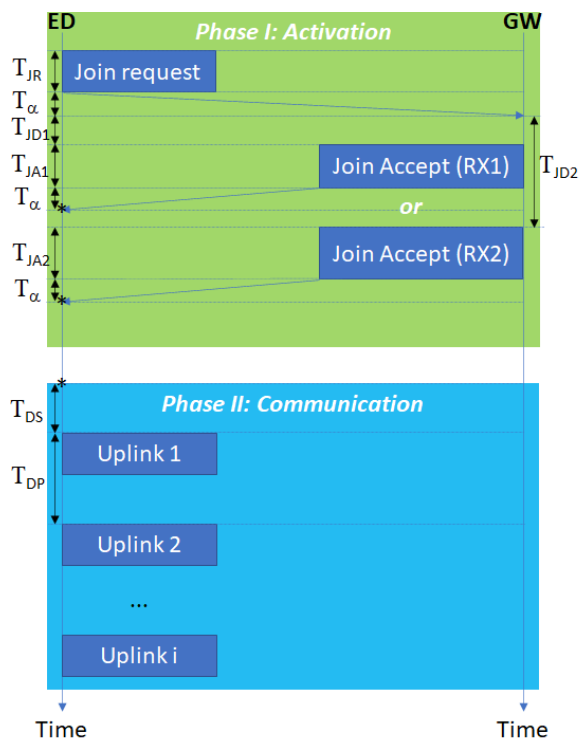

(a)

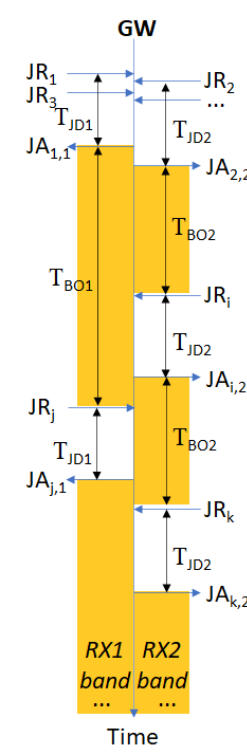

(b)
Fig. 5. Identified problem (a) phases of LoRaWAN operation for a single $\mathrm{ED}$, (b) timing for OTAA for multiple EDs on a GW

the backoff time for these packets calculated using 7 for most common DC limits (refer to Table I).

\section{Considered Scenario AND Problem IDENTIFICATION}

Consider multiple LoRaWAN ED desiring to connect to a network. Without the loss of generality, we imply that: (i) only a single GW is present, (ii) the network is configured to use only three default channels (belonging to a single subband) for UL communication and a dedicated channel for RX2, (iii) all EDs use the same SF, and (iv) the EDs are not synchronized, their JR transmissions are periodic, and their starting times are distributed uniformly in time. In practice, the described situation is very likely, e.g., in the case, if the EDs have been deployed before the GW/NS, after a GW/NS has been replaced or has been out-of-service for a decently long time (thus the EDs have detected this and switched back to connection reestablishment phase).
Following the procedures discussed in the previous subsections, after receiving the first JR, the GW replies with a JA and backoffs future transmission in this band for a specified amount of time. The ED, given that it has correctly received the JA, switches to its normal operation mode conveying application-layer data. The whole procedure is illustrated in Fig. 5 (a). After the backoff time expires, the GW responds to the next JA it receives. One can see that while the number of the EDs attempting to join the network is big and thus the number of JR received in a unit of time is small, the new EDs will be admitted to the network almost periodically - with a period slightly exceeding the sum of the respective JOIN_ACCEPT_DELAY and the backoff duration after the JR packet (illustrated in Fig. 5 (b); alternatively, in the case if a check for channel availability is handled at RX1 or RX2 transmission opportunity the delay between EDs admittance equals the minimum period of JR packet, accounting for the DC). As this has been already discussed, a real-life LoRaWAN network operates using at least two RX windows configured to the different frequency subbands. Thus one will observe two such periodic processes - as this is shown in Fig. 5 (b).

Next, we recall that the versatile monitoring applications represent the primary use case for LoRaWAN, which means that the UL reports of real-life sensor-EDs are very often periodical [16]. When attempting to connect to the network, the real-life EDs also often attempt JRs periodically (we have observed this behaviour, e.g., for [17] sensors). The delay between JA reception and sending a UL, based on our experience with the real-life sensors, is also typically kept as small as possible. Given all these facts, we come up with the hypotheses, which we intend to evaluate through simulations in the following section of the paper: even if the starting time of ED operation in a dense LoRaWAN networks are distributed uniformly in time, the specifics of OTAA activation may result in non-uniform distribution of the periodic UL packet transmission.

\section{Validation Methodology and Numeric Results}

\section{A. Validation Methodology and Developed Simulator Model}

To validate our hypothesis, we have developed a MATLABbased simulation model (can be downloaded from [3]) modelling in details the OTAA procedure with a consequent data communication. The model simulates a single LoRaWAN GW's operation, and multiple EDs deployed around it. For tractability, we imply that all the EDs are configured to use the same SF and can reach the GW, and that all packets, colliding in time and frequency channel, get lost. The developed model allows modifying the number of EDs, their traffic patterns (both for JA and consequent UL data traffic) and packet sizes, the number and DC of UL/DL frequency channels, and the SF employed by EDs and the GW. The key parameters configured for our models are summarized in Table III.

To validate the correctness of the developed simulation model, its results have been compared against of the analytic models for networks based on unslotted Aloha with single and multiple frequency channels (equation (3) in [18]). During 


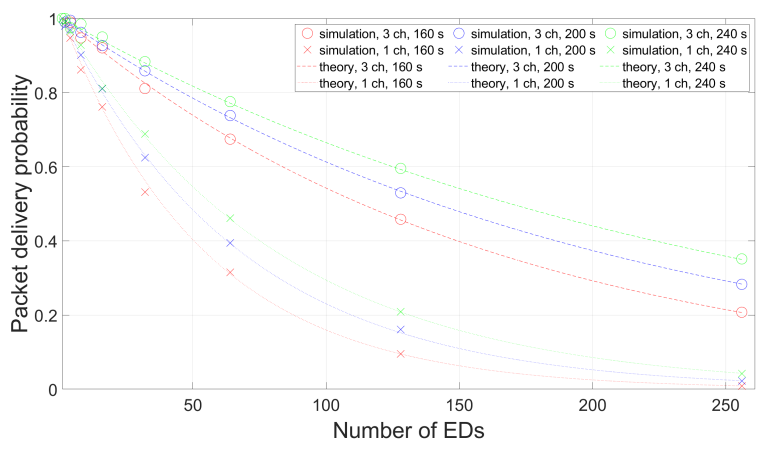

Fig. 6. Probability of packet delivery versus the number of EDs: theory and simulation results (average over 100 iterations, packet duration $1.483 \mathrm{~s}$, packet periods 160,200 or $240 \mathrm{~s}$ ).

TABLE III

KEY PARAMETERS FOR SIMULATIONS

\begin{tabular}{|c|c|c|}
\hline Parameter & Value & Unit \\
\hline$R E C E I V E \_D E L A Y 1$ & 1 & $\mathrm{~s}$ \\
\hline$R E C E I V E \_D E L A Y 2$ & 2 & $\mathrm{~s}$ \\
\hline JOIN_ACCEPT_DELAY1 (T $\left.T_{J D 1}\right)$ & 5 & $\mathrm{~s}$ \\
\hline JOIN_ACCEPT_DELAY2 (T $\left.T_{J D 2}\right)$ & 6 & $\mathrm{~s}$ \\
\hline$D R_{J A}$ and $D R_{U L}$ & 0 & - \\
\hline$R X 1 D R o f f s e t$ & 0 & - \\
\hline UL and RX1 number of channels & 3 & - \\
\hline $\mathrm{UL}$ and $\mathrm{RX} 1$ subband & h1.5 & - \\
\hline UL and RX1 subband DC & 1 & $\%$ \\
\hline RX2 subband & h1.7 & - \\
\hline RX2 DC & 10 & $\%$ \\
\hline$T_{J A}$ & 200 & $\mathrm{~s}$ \\
\hline$T_{U L}$ & $160 . .192$ & $\mathrm{~s}$ \\
\hline$N_{E D}$ & $1 . .512$ & - \\
\hline$T_{\text {sim }}$ & 4 & hours \\
\hline iterations & TBD & - \\
\hline$B_{\mathrm{JA}}=B_{\mathrm{JA}(\mathrm{CFList})}$ & 29 & byte \\
\hline$B_{\mathrm{App}}$ & 9 & byte \\
\hline
\end{tabular}

these tests, the simulation of the OTAA procedure have been disabled, and the transmissions of the EDs have been distributed uniformly in time. The results presented in Fig. 6 show an accurate match between the developed simulator and the theory, thus confirming that the collisions by the developed simulator are modelled correctly.

Next, we proceeded with simulating the OTAA and subsequent data communication. We imply that the initial JR transmissions of EDs are uniformly distributed in time within the period of 0 to $T_{J R}$ seconds. In the case, if an ED does not receive a JA in RX1 or RX2, it retransmits the next $\mathrm{JR}$ after $T_{J R}$. After receiving the JA, an ED immediately switches to normal communication mode implying periodic packet transmission with a period of $T_{U L}$. In the case, if an ED or the GW cannot send the packet due to DC restrictions, the packet is discarded. When scheduling a DL, the GW prioritizes the use of RX1 to RX2. For each set of parameters, we have run the simulation 100 times with further statistical processing to calculate the minimum, maximum and mean values. Each run implied simulation of 4 hours of the network's operation.

\section{B. Numeric Results}

Figure 7 demonstrates the increase in the number of EDs connected to the network in time. One can see that when the number of devices is decently big, the curve's initial section has a linear trend. For example, for 256 EDs by 1986 seconds,

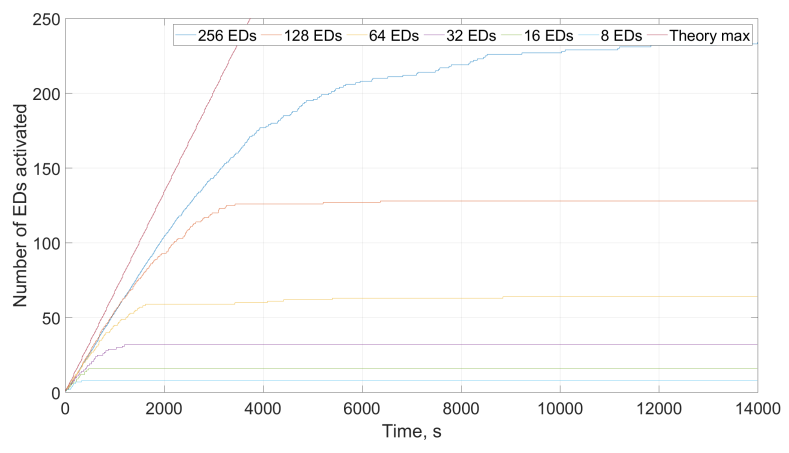

Fig. 7. Number of EDs accepted to the network versus time from the start of simulation for different number of EDs attempting to join $\left(T_{U L}=164 \mathrm{~s}\right)$.

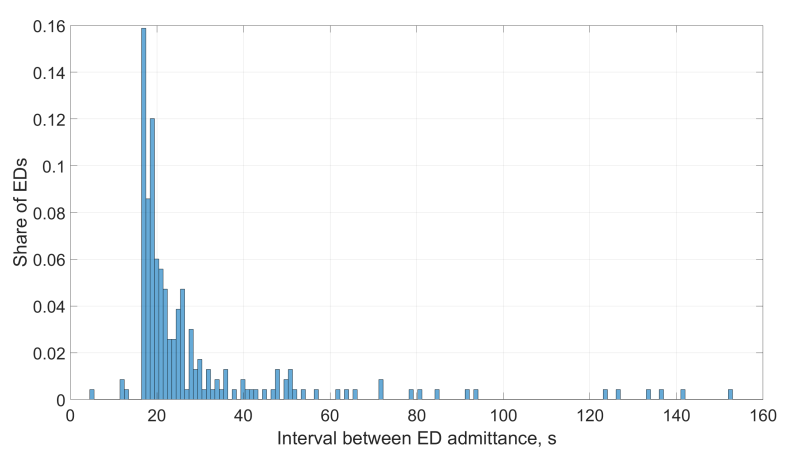

Fig. 8. Distribution of the time period between sequential EDs acceptance $\left(T_{U L}=164 s ; 1 \mathrm{~ms}\right.$ bins, $\left.256 \mathrm{EDs}\right)$.

104 EDs have joined the network (one device joining every 19.1 seconds on average). In theory, imposing configurations listed in III, a LoRaWAN GW may accept nodes at minimum every $16.45 /(1+0.1)=14.95$ (refer to Table II and note presence of both RX1 and RX2 with respective DC; the respective line, labelled "Theory max", is depicted on Figure 7 for reference). However, since some of the JR and JA packets in RX1 are lost due to collisions, and the fact that due to the finite number of EDs there is a small delay between backoff expiration and scheduling a next JR, the observed rate of device connection is lower than the theoretical maximum. With the increase of time and the number of EDs already in the network, the trend becomes sublinear due to the increase of the periods between the JR. A similar effect can be seen when the number of simulated EDs becomes lower. This is also worth noting that not all of the 256 EDs have managed to activate in the network by the end of the simulation.

Figure 8 illustrates the distribution of the periods between sequential activation of the two EDs for one single simulation run. From the presented results one can see that in about 36\% of the cases the next ED joins the network within 16.5-19.5 seconds from a previous ED, and in $60 \%$ of the cases - within 16.5-23.5 seconds interval. Note, that the values of below 16.5 seconds correspond to the case when the different RX windows (i.e., RX1 and RX2) have been used to deliver the two sequential JRs.

Finally, Figure 9 illustrates the distribution of the number of UL data transmissions in time within one $T_{U L}$ period during the actual data communication phase. One can see the non- 


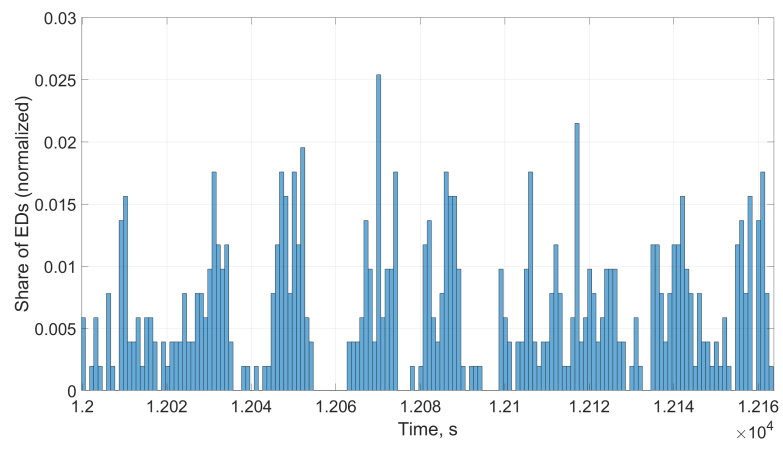

Fig. 9. Distribution of UL data transmissions in time $\left(T_{U L}=164 \mathrm{~s}, 512\right.$ EDs).

uniform distribution of the UL packets in time and the periodic (with a period of about 17 seconds) fluctuation of the number of EDs sending their data.

\section{CONCLUSIONS}

The LPWANs in general and LoRaWAN, in particular, has today become one of the critical elements for connecting and collecting data from the IoT deployments. However, there are still effects which are neither known nor accounted for. One of these, focused on in this study, is the non-uniform uplink traffic distribution in time in LoRaWAN networks. Initially, we have observed this effect in the multi-hundred ED reallife LoRaWAN network deployed as a collaboration between 5GTN and Smart Campus initiative in the University of Oulu. In this study, we focus on identifying the possible reasons, which may have caused such behaviour.

Specifically, we hypothesized that this effect could be caused by the operation of OTAA under DC limits and, specifically, the way the real-life LoRaWAN devices manage the DC limitations. To validate this hypothesis, we have (i) developed the MATLAB-based simulation mode, (ii) validated it, and (iii) conducted a set of simulations. The obtained results confirm our hypothesis and show that if a significant number of LoRaWAN EDs attempt to join the network using OTAA within a short period of time - they will be admitted to the network periodically. This will result, in the case the UL traffic of these devices is periodic (which seems to be true for many real-life LoRaWAN sensors), in the non-uniform distribution of UL packets transmissions in time, thus causing extra packet losses during the most "crowded" periods. To the best of our knowledge, neither this effect (except for [2] itself) nor the explanation for it has been offered by the previous studies.

Given the complex nature of the observed effect, in this work, we limited our discussion to identifying and detailing its background and confirming the correctness of our hypothesis regarding the combination of the mechanisms and preconditions, which cause it. However, the next step, which we leave primarily for further studies, is the identification of effective ways to mitigate the identified problem. For this, we consider the following approaches and mechanisms to be possible:

- Introduction of a random/pseudo-random delay between JA and the first UL packet and/or introducing a random component in the UL periodic traffic. Note, that this solution neither implies any modification of the current LoRaWAN specification, nor the introduction of the novel LoRaWAN communication procedures.

- Introduction of a novel DL command, allowing the NS to re-schedule (e.g., by introducing a specified or a random delay) the periodic UL transmissions of an ED, as well as the development of specific algorithms running at NS or GW for detecting the non-uniform distribution of UL packets in time.

In the further works, we intend to evaluate the performance of these approaches and study the effect of the various other aspects (e.g., non-homogeneous network nature, different EDs traffic patterns, multi-GW scenarios, capture effect) on the identified effect.

\section{ACKNOWLEDGMENT}

This research has been financially supported by the Academy of Finland 6Genesis Flagship (decision 318927) and MRAT-SafeDrone (decision 341111) project.

\section{REFERENCES}

[1] Statista, "Number of LPWAN connections by technology worldwide from 2017 to 2023," 2021. [Online]. Available: https://www.statista.com/statistics/880822/lpwan-ic-market-shareby-technology/

[2] R. Yasmin, K. Mikhaylov, and A. Pouttu, "LoRaWAN for Smart Campus: Deployment and Long-Term Operation Analysis," Sensors, vol. 20, no. $23,2020$.

[3] K. Mikhaylov, "GitHub repository: LoRaWAN OTAA model," 2021. [Online]. Available: https://github.com/kvmikhayl/LoRaWAN_OTAA

[4] N. Sornin and A. Yegin, Eds., "LoRaWAN 1.1 Regional Parameters," 2017.

[5] B. S. Chaudhari and M. Zennaro, Eds., LPWAN Technologies for IoT and M2M Applications. U.K.: Academic Press, 2020.

[6] S. Kim, L. Heonkook, and S. Jeon, "An Adaptive Spreading Factor Selection Scheme for a Single Channel LoRa Modem," Sensors, vol. 20, no. 4, 2020.

[7] D. Croce, M. Gucciardo, S. Mangione, G. Santaromita, and I. Tinnirello, "Impact of LoRa imperfect orthogonality: Analysis of link-level performance," IEEE Communications Letters, vol. 22, no. 4, pp. 796-799, 2018.

[8] R. Kufakunesu, G. Hancke, A. Abu-Mahfouz, and M. Adnan, "A Survey on Adaptive Data Rate Optimization in LoRaWAN: Recent Solutions and Major Challenges," Sensors, vol. 20, no. 19, 2020.

[9] Semtech Corporation, "AN1200.13 - SX1272/3/6/7/8: LoRa Modem Designer's Guide ," 2013.

[10] L. Casals, B. Mir, R. Vidal, and C. Gomez, "Modeling the Energy Performance of LoRaWAN," Sensors, vol. 17, 2017.

[11] N. Sornin and A. Yegin, Eds., "LoRaWAN 1.1 Specification," 2017.

[12] LoRaWAN Alliance, "LoRaWAN Security: Frequently Asked Questions," 2016.

[13] Semtech, "TN1300.01 - How to Qualify a LoRaWAN Device in Europe," 2018.

[14] CEPT ECC, "ERC Recommendation 70-03," 2020.

[15] ETSI, "ETSI TR 103526 V1.1.1: System Reference document," 2018.

[16] H. Rajab, T. Cinkler, and T. Bouguera, "IoT scheduling for higher throughput and lower transmission power," Wireless Netw., vol. 2020, 2020.

[17] Elsys, "ERS,” 2021. [Online]. Available: https://www.elsys.se/en/ers/

[18] C. Goursaud and Y. Mo, "Random Unslotted Time-Frequency ALOHA: Theory and Application to IoT UNB Networks," in Proc. 23rd Int. Conf. Telecommun., 2016, pp. 1-5. 